\title{
SPECTROSCOPY
}

\section{Probing phonons}

Dielectric gaps between closely spaced metal particles can result in large enhancements of electromagnetic fields. This effect is often used for increasing the output signal from nonlinear optical processes such as Raman scattering, which depends on the fourth power of the electric field. Daniel Sigle, Jeremy Baumberg and colleagues in the United Kingdom and France have used surface-enhanced Raman scattering (SERS) to investigate the phonon modes of individual ultrathin $\mathrm{CdSe}$ nanoplatelets (Phys. Rev. Lett. 113, 087402; 2014). The platelets were fabricated in a nanogap between a 70-nm-thick gold film and a gold particle with a diameter of $100 \mathrm{~nm}$. Varying the thickness of the CdSe plates in the nanogaps modified the Raman shift and enabled analysis of the highwavenumber phonon dispersion.

"The SERS spectra, taken on single nanoplatelets sandwiched between a gold nanoparticle and a gold surface, reveal a phonon doublet arising from oscillations perpendicular to and within the platelet plane," Baumberg told Nature Photonics. "The out-of-plane mode cannot be observed with conventional Raman spectroscopy. The resulting strong electric field enhancements

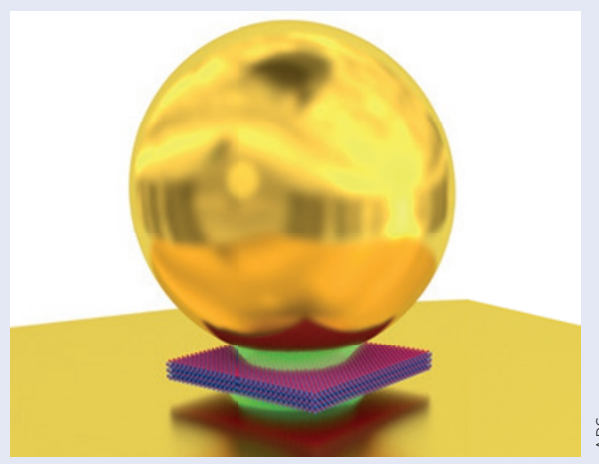

and the field vector reorientation within such nanometre-sized plasmonic gaps reveal otherwise hidden information deep into the Brillouin zone, illuminating the vibrational properties of ultrathin materials."

When asked about how they came to the result, Baumberg told Nature Photonics that when they realized they could put very thin semiconductors in a nanogap between these metal structures, they wanted to couple the excitonic transition to the confined plasmon. Baumberg's group has worked for some time on developing a mechanistically relevant strongly-coupled semiconductor microcavity system. In the early stages of their work with $\mathrm{CdSe}$ they used SERS to verify the quality of the CdSe in the nanogap and noticed the unusual modification of the Raman shift.

According to Baumberg, the work was possible thanks to collaboration with a French CNRS group that specializes in growing these platelets with a specific thickness of atomic layers, as well as developing the plasmon nanostructures that allow the CdSe to be trapped in such tiny cavities. Apart from fabrication, another challenge is collecting enough signal from the nanojunction to see the CdSe over time, but at the same time avoiding destroying the semiconductor with too intense illumination.

The work shows that by putting semiconductors into plasmonic nanocavities it is possible to observe new properties and break the normal selection rules. The hope is that this work has implications for the new generation of two-dimensional materials such as graphene. The team hopes to be able to directly watch electron interactions and control them to create ultra-low-power devices. Towards this goal, Baumberg says they have been splitting water using such small nanocavities.

\section{DAVID PILE}

\section{VIEW FROM... PHOTON14}

\section{Taking the quantum leap}

London hosted this year's Photon conference. Quantum optics and improved technology commercialization in the UK were two key points of discussion.

\section{Maria Maragkou}

uantum physics was the toast of the town during Photon14, which was held at Imperial College in London over 1-4 September. The event organized by the UK's Institute of Physics (IOP) - was the 8 th in a conference series that started in 2002 and aims to bring together researchers with a common interest in optics and photonics.

The first plenary session was given by former IOP President Peter Knight and dedicated to the future of photonics in the UK, which has taken an unexpected upturn following the UK government's announcement at the start of 2014 of a colossal investment of more than $£ 200$ million in quantum technology.

In February, the Engineering and Physical Sciences Research Council (EPSRC), the UK's main funding body for university research in physics and engineering, opened a call for a nationwide network of Quantum Technology Hubs supported by a $£ 155$ million fund. Such actions have spurred enthusiasm among the quantum physics community and with good reason; quantum research in the UK has strengthened over the years with numerous groups all over the country active in theoretical and applied aspects of quantum physics. This was also reflected in the conference program: almost onequarter of the sessions were devoted to quantum information, quantum optics or quantum metrology. Among many beautiful talks, Mauro Paternostro from Queen's University in Belfast discussed a way of assessing the non-equilibrium thermodynamics in a quenched quantum many-body system. In some of the most crowded sessions of the conference, Ted Santana from Heriot-Watt University 